\title{
Clinical Response, Outbreak Investigation, and Epidemiology of the Fungal Meningitis Epidemic in the United States: Systematic Review
}

Kaja M. Abbas, PhD; Nargesalsadat Dorratoltaj, MPH; Margaret L. O'Dell, MD; Paige Bordwine, MPH; Thomas M. Kerkering, MD; and Kerry J. Redican, PhD

\section{ABSTRACT}

We conducted a systematic review of the 2012-2013 multistate fungal meningitis epidemic in the United States from the perspectives of clinical response, outbreak investigation, and epidemiology. Articles focused on clinical response, outbreak investigation, and epidemiology were included, whereas articles focused on compounding pharmacies, legislation and litigation, diagnostics, microbiology, and pathogenesis were excluded. We reviewed 19 articles by use of the PRISMA (Preferred Reporting Items for Systematic Reviews and Meta-Analyses) framework. The source of the fungal meningitis outbreak was traced to the New England Compounding Center in Massachusetts, where injectable methylprednisolone acetate products were contaminated with the predominant pathogen, Exserohilum rostratum. As of October 23, 2013, the final case count stood at 751 patients and 64 deaths, and no additional cases are anticipated. The multisectoral public health response to the fungal meningitis epidemic from the hospitals, clinics, pharmacies, and the public health system at the local, state, and federal levels led to an efficient epidemiological investigation to trace the outbreak source and rapid implementation of multiple response plans. This systematic review reaffirms the effective execution of a multisectoral public health response and efficient delivery of the core functions of public health assessment, policy development, and service assurances to improve population health. (Disaster Med Public Health Preparedness. 2016;10:145-151)

Key Words: communicable diseases, disease outbreaks, epidemics, infection control, public health practice

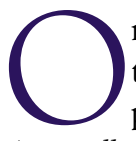
n September 18, 2012, a clinician reported to the Tennessee Department of Health a patient diagnosed with culture-confirmed Aspergillus fumigatus meningitis, 46 days after an epidural steroid injection. ${ }^{1,2}$ This patient was the index case of the 2012-2013 multistate fungal meningitis epidemic in the United States.

The source of the fungal meningitis epidemic was traced to contaminated lots of preservative-free methylprednisolone acetate that had been used for epidural steroid injections and had been produced at the New England Compounding Center in Massachusetts. ${ }^{3}$ The New England Compounding Center recalled injectable methylprednisolone acetate products, which eventually expanded to all drug products, and completely ceased drug production.

The timeline of the fungal meningitis outbreak response is illustrated in Figure 1. Contaminated lots of preservative-free methylprednisolone acetate were used in epidural steroid injections from July 30, 2012, to
September 18, 2012, until the first case of fungal meningitis was confirmed in Tennessee on September 18, 2012. A total of 23 states had received contaminated lots of preservative-free methylprednisolone acetate from July 30, 2012, to September 18, 2012. The Centers for Disease Control and Prevention (CDC) released interim treatment guidelines for fungal meningitis on October 19, 2012, which recommended voriconazole therapy, which was prescribed for all patients in Virginia and other affected states. Michigan performed follow-up magnetic resonance imaging (MRI) on patients for 6 months until April 2013. The final case count stood at 751 patients and 64 deaths, and 20 affected states, as of October 23, 2013, and no additional cases are anticipated.

The fungal meningitis epidemic was controlled through a multisectoral response from the affected patients, immediate caregivers of family and friends, hospitals and pharmacies, and public health departments at the local, state, and federal levels, as illustrated in Figure 2. Clinics and local health departments 
FIGURE

Timeline of the Fungal Meningitis Outbreak. The first case was confirmed in Tennessee on September 18, 2012. Twenty-three states had received contaminated lots of preservative-free methylprednisolone acetate, and 20 states reported confirmed cases of fungal meningitis. As of October 23, 2013, the case count stood at 751 patients and 64 deaths, and no additional cases are anticipated. Abbreviation: MMWR, Morbidity and Mortality Weekly Report.
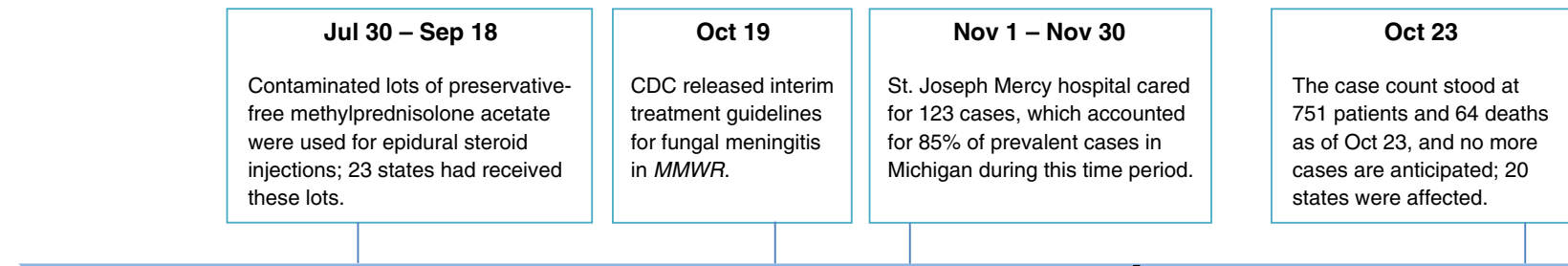

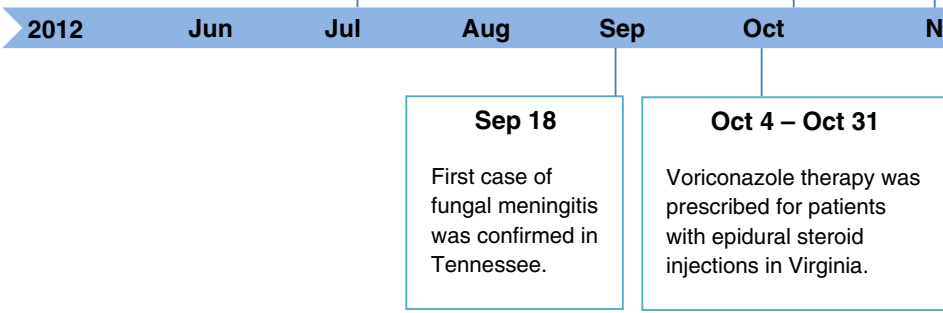

\begin{tabular}{|l|}
\hline \multicolumn{1}{|c|}{2013} \\
Nov 9 - Apr 30 \\
MRI screenings were performed on \\
172 patients of St. Joseph Mercy \\
hospital in Michigan.
\end{tabular}

contacted the patients who had received the contaminated epidural steroid injections and referred probable cases for clinical services and hospitalization. Patients exposed to fungal-contaminated epidural injections were followed up for 6 months and were provided appropriate treatment including lab tests and hospitalizations.

The objective of the present study was to conduct a systematic review of the multistate fungal meningitis outbreak response in the United States, with a focus on clinical response, outbreak investigation, and epidemiology. We focused this systematic review on the coordinated response and partnership between the clinical system and the public health departments at the local, state, and federal levels to understand the public health assessment, policy development, and service assurances in control and prevention of the fungal meningitis epidemic. Systematic understanding of the multisectoral outbreak response to the fungal meningitis epidemic will assist in analyzing this response and improve the emergency preparedness of the health departments to prevent and control future outbreaks effectively and efficiently. The public health significance of this systematic review of the fungal meningitis epidemic is to analyze the implementation of the public health core responsibilities of assessment, policy development, assurance, and related essential services in protecting the health of the public.

\section{SYSTEMATIC REVIEW}

\section{Search Strategy}

We searched the PubMed database for articles published from September 1, 2012, to April 30, 2015, with the terms ("meningitis, fungal" or ["meningitis" and "fungal"] or "fungal meningitis" or ["fungal" and "meningitis"]) and ("disease outbreaks" or ["disease" and "outbreaks"] or "disease outbreaks" or "outbreak").

\section{Data Abstraction and Synthesis}

The data abstraction and synthesis process was conducted by 2 authors (KMA and ND) independently. We resolved discordant decisions through consensus. Data abstraction and synthesis included the following 4 steps: identification, screening, eligibility, and inclusion. During the identification step, articles were identified by using the search strategy mentioned above. During the screening step, duplicate articles were removed, and titles and abstracts of the remaining articles were screened to determine their relevance to our study objectives. During the eligibility step, the full text of the articles was analyzed to determine their relevance to our study objectives.

\section{Inclusion and Exclusion Criteria}

The inclusion criteria were articles focused on clinical response, outbreak investigation, and epidemiology. Exclusion criteria were articles focused on compounding pharmacies, legislation and litigation, diagnostics, microbiology, pathogenesis, press releases, editorials, and other diseases or outbreaks.

\section{PRISMA Process}

Figure 3 illustrates the process flow diagram of identification, screening, eligibility, and inclusion of articles for the systematic review by use of the PRISMA (Preferred Reporting Items for Systematic Reviews and Meta-Analyses) flow diagram. ${ }^{4}$ 
Multisectoral Public Health Response. The fungal meningitis epidemic was controlled through a multisectoral response from the affected patients, immediate caregivers of family and friends, hospitals and pharmacies, and public health departments at the local, state, and federal levels. Abbreviations: CDC, Centers for Disease Control and Prevention; FDA, Food and Drug Administration.

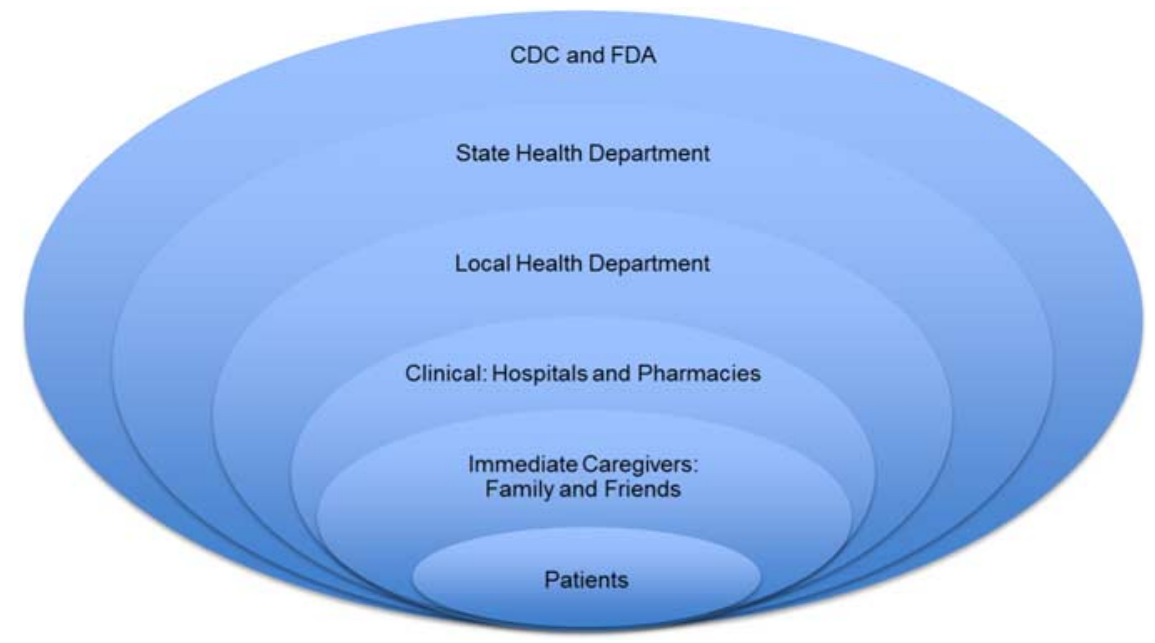

Nineteen articles met the selection criteria for the systematic review of the fungal meningitis epidemic. Articles were categorized by clinical response, outbreak investigation, and epidemiology.

\section{CLINICAL RESPONSE}

The clinical response to the fungal meningitis epidemic is summarized in Table 1.

\section{Interim Treatment Guidelines}

The CDC released interim guidelines for fungal meningitis treatment in the Morbidity and Mortality Weekly Report on October 19, 2012. Voriconazole was recommended as effective in treating infections caused by the predominant pathogen, Exserohilum rostratum, in this outbreak. ${ }^{3}$

\section{Evolving Treatment Guidelines}

The fungal meningitis patients presented with a broad spectrum of clinical disease, reflecting the differences in pathogenesis, exposure, and host risk factors. Treatment guidelines issued by the CDC initially recommended high-dose dual therapy with voriconazole and liposomal amphotericin B, which was revised to monotherapy with voriconazole for patients without serious illness. Treatment recommendations were updated as the fungal meningitis outbreak evolved as new clinical evidence emerged during therapeutic management of affected patients. ${ }^{5}$ More than $90 \%$ of patients at risk of developing fungal infection following the contaminated epidural injections were asymptomatic and did not develop any infection. Pharmacies involved in the outbreak response were challenged by both clinical and administrative issues while adapting to the evolving treatment guidelines from the CDC. ${ }^{6}$

\section{Voriconazole Therapy}

On the basis of a case series analysis of 172 patients who had epidural steroid injections and presented for care at a hospital in Virginia from October 4, 2012, to October 31, 2012, voriconazole therapy was prescribed for all patients. Meningitis was diagnosed in most of these patients, and the voriconazole therapy improved prognosis within the treated patient cohort.

\section{Michigan Hospital}

In November 2012, St. Joseph Mercy Ann Arbor hospital cared for 123 patients or $85 \%$ of the cases in Michigan, which related to $25 \%$ of the total cases nationally during this time. ${ }^{8}$ A total of 620 patients had received contaminated steroid injections from the same pain clinic located 26 miles from the hospital. The high proportion of infections from this clinic was due to "hot lots" of steroids received at this clinic; these drug lots were associated with a large number of infections. The Saint Joseph Mercy hospital identified a cluster of fungal meningitis cases immediately after the onset of the outbreak in Tennessee. The treatment familiarity led to a centralized approach of consistent care delivery and care management at Saint Joseph Mercy hospital, which became the default health facility for the infected patients in Michigan to seek treatment. Owing to the long-term uncertainty about the natural progression of fungal meningitis, it was recommended 
FIGURE 3

PRISMA Flowchart. The Preferred Reporting Items for Systematic Reviews and Meta-Analyses (PRISMA) flow diagram of articles' identification, screening, eligibility, and inclusion in the systematic review is illustrated. Articles focused on clinical response, outbreak investigation, and epidemiology were included, whereas articles focused on compounding pharmacies, legislation and litigation, diagnostics, microbiology, pathogenesis, press releases, editorials, and other diseases or outbreaks were excluded.
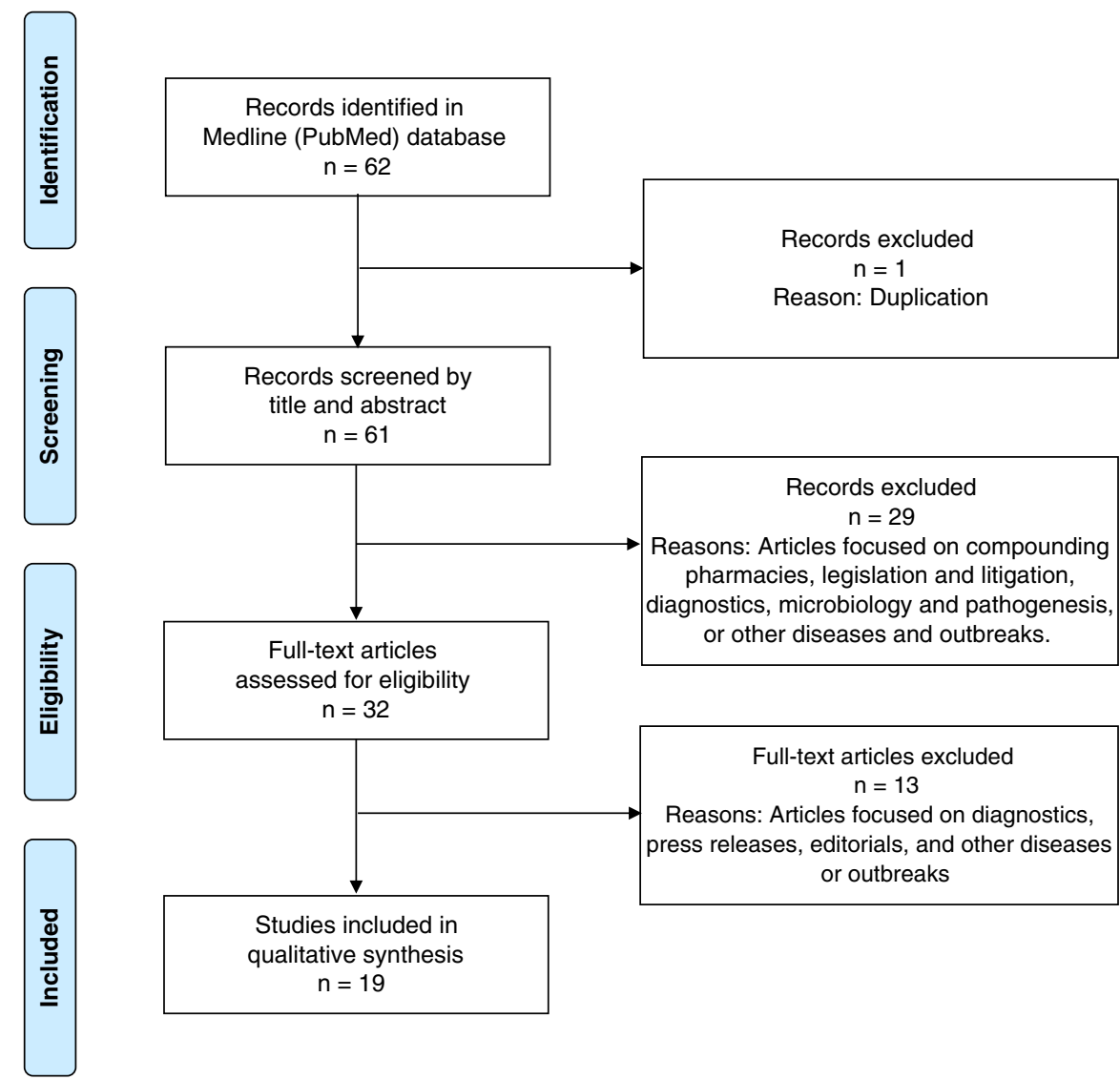

that patients continue oral voriconazole treatment at home following hospital release for months based on the follow-up tests and disease progression. ${ }^{9}$ The patients were closely monitored for optimal drug intake and any adverse side effects. MRI was performed on 172 patients between November 9, 2012, and April 30, 2013; these patients had received "hot lot" steroids but had not presented for clinical care for treatment of adverse effects. ${ }^{10}$ Of these patients, $21 \%$ had abnormal MRI findings and were initiated on early medical or surgical treatment for spinal infections. As of January 31, 2014, a total of 153 patients had been diagnosed at Saint Joseph Mercy hospital for probable or confirmed spinal or paraspinal infection at the injection site, of which 78 patients were confirmed to have fungal infection. Voriconazole was prescribed as the drug therapy for all 153 patients, among which 115 patients were also prescribed liposomal amphotericin B. ${ }^{11}$ A total of 20 patients remained on an azole agent as of January 31, 2014.

\section{Risk of Fungal Infection}

Pappas reviewed the risk of developing fungal infection following an injection of contaminated methylprednisolone acetate. ${ }^{12}$ Less than $10 \%$ of patients at risk developed any symptoms of infection, and among symptomatic patients, some developed meningitis while others developed epidural abscess and other parameningeal complications. There was no consensus in the management of exposed asymptomatic patients and symptomatic patients with negative lab test results, such as cerebrospinal fluid, glucose, protein, and imaging tests. Thereby, there were variations in the clinical treatment of exposed patients who were symptomatic or asymptomatic.

\section{OUTBREAK INVESTIGATION}

The outbreak investigation of the fungal meningitis epidemic is summarized in Table 2. 


\section{Clinical Response to the Fungal Meningitis Epidemic ${ }^{a}$}

\section{Reference Inferences}

$3 \quad$ CDC recommends voriconazole as an effective drug to treat infections caused by the predominant pathogen, Exserohilum rostratum.

5,6 Pharmacies adapted to the emerging clinical evidence and evolving treatment recommendations from the CDC; high-dose dual therapy with voriconazole and liposomal amphotericin B was revised to monotherapy with voriconazole for patients without serious illness.

$7 \quad 172$ patients were exposed to contaminated epidural steroid injections and presented for care in a Virginia hospital; most of these patients were diagnosed with meningitis and most patients improved their health with voriconazole therapy.

$8 \quad$ Fungal meningitis treatment familiarity at Saint Joseph Mercy hospital led to a centralized approach of consistent care delivery and care management for the infected patients in Michigan.

The novelty of the fungal meningitis infections led to long-term uncertainty about their clinical progression, and patients were closely monitored for optimal drug intake and any adverse effects.

Patients were recommended to continue oral voriconazole treatment at home, following hospital release for months based on the follow-up tests and disease progression.

Magnetic resonance imaging screenings assisted in identifying patients for initiating early medical or surgical treatment.

Voriconazole drug therapy was prescribed for all 153 patients with probable or confirmed spinal or paraspinal infection at the Saint Joseph Mercy hospital in Michigan, with liposomal amphotericin B also prescribed for 75\% of these patients.

There is no consensus in management of exposed asymptomatic patients and symptomatic patients with negative lab tests, thereby leading to variations in clinical treatment of exposed patients, both symptomatic and asymptomatic.

${ }^{a}$ Abbreviation: CDC, Centers for Disease Control and Prevention.

\section{TABLE 2}

\section{Outbreak Investigation of the Fungal Meningitis Epidemic}

\section{Reference Inferences}

13 Collaboration between the public health system and essential health care partners led to an effective outbreak response, including the detection of infection cluster to the rare pathogen, followed by rapid implementation of multiple response plans.

14 Good collaboration between the public health and clinical systems in controlling the fungal meningitis outbreak facilitated the fast-moving epidemiological investigation to rapidly trace the outbreak to its source.

15 A hybrid active public health surveillance system was implemented by the Virginia Department of Health, in partnership with clinical stakeholders, for control and prevention of the fungal meningitis outbreak in Virginia.

\section{Public Health Preparedness}

Bell and Khabbaz illustrate the value of public health preparedness in detection and response to emerging infections. ${ }^{13}$ During the fungal meningitis outbreak, collaboration between the public health system with essential health care partners in the private sector, industry, and academia enabled effective control and prevention interventions. The outbreak response included detection of an infection cluster to the rare pathogen, followed by rapid implementation of multiple response plans, which led to a decrease in the case fatality rate among the fungal meningitis patients.

\section{Multisectoral Response}

The public health system of CDC (in collaboration with the Food and Drug Administration), state and local health departments, along with the clinical system of clinics, hospitals, and pharmacies collaborated well in prevention and control of the fungal meningitis outbreak. ${ }^{14}$ While the clinical system identified and treated the patients to control the rare invasive fungal infection, the public health system conducted a rapid epidemiological investigation to trace the source of the outbreak to the New England Compounding Center to prevent additional fungal infections. The New England Compounding Center responded by recalling all injectable methylprednisolone acetate products, which eventually expanded to all drug products and a complete ceasing of drug production.

\section{Hybrid Surveillance Strategy}

The Virginia Department of Health implemented a hybrid active public health surveillance system for control and prevention of the fungal meningitis outbreak in Virginia, in partnership with clinical stakeholders. ${ }^{15}$ Division of labor was coordinated to improve efficiency of the outbreak response, with clinicians focused on diagnosis and treatment, while the health departments undertook weekly surveillance phone calls to exposed patients who were not in clinical care.

\section{EPIDEMIOLOGY}

The epidemiology of the fungal meningitis epidemic is summarized in Table 3. 
TABLE 3

Epidemiology of the Fungal Meningitis Epidemic

Reference Inferences

16 The fungal meningitis cases presented a broad spectrum of clinical disease, reflecting the differences in pathogenesis, exposure, and host risk factors. 2 Cohort analysis on patients who had been given epidural or paraspinal glucocorticoid injections in Tennessee showed that the infection risk was high among patients exposed to lot 06292012@26, older vials, higher doses, multiple procedures and in patients in whom a translaminar approach to epidural glucocorticoid injection was used.

17

As of July 1, 2013, morbidity and mortality rates of the multistate fungal meningitis outbreak were estimated at 749 reported cases with 61 deaths (8\%); 40 patients (5\%) had stroke, and 229 (31\%) patients had meningitis.

1,18-20 As of October 23, 2013, the multistate fungal meningitis outbreak had affected 20 states and the case count stood at 751 patients with 64 deaths, with no additional cases anticipated. A total of 233 patients had meningitis only, 325 patients had paraspinal/spinal infection only, 33 patients had peripheral joint infection only, 151 patients had meningitis and paraspinal/spinal infection, 2 patients had paraspinal/spinal infection and peripheral joint infection, and 7 patients had stroke.

\section{Six states: Florida, Indiana, Michigan, New Jersey, Tennessee, and Virginia}

Chiller et al reviewed the case records of 328 patients from the 6 states (Florida, Indiana, Michigan, New Jersey, Tennessee, Virginia) with the most reported cases through November 19, $2012 .{ }^{16}$ On the basis of their initial clinical findings, they concluded that the fungal infections during this outbreak caused a broad spectrum of clinical disease, reflecting the differences in pathogenesis, exposure route, and host risk factors.

\section{Clinic: Tennessee}

Kainer et al conducted a cohort analysis at both the patient and procedure levels on all patients who had been given epidural or paraspinal glucocorticoid injections at a single clinic in Tennessee since July 1, 2012, to evaluate risk factors for infection. ${ }^{2}$ Cases were defined as patients who developed fungal meningitis, posterior circulation stroke, spinal osteomyelitis, or epidural abscess after injection. The median age of the cases was 69 years (range, 23-91 years). The median time from the last injection to symptom onset was 18 days (range, $0-56$ days), with meningitis alone among $73 \%$ of patients. A total of 22 patients were confirmed by lab tests to have Exserohilum rostratum (21 patients) and Aspergillus fumigatus (1 patient) infections; 8 patients died, of which 7 deaths were due to stroke. The infection risk was high among patients exposed to lot number 06292012@26, older vials, higher doses, and multiple procedures and in patients in whom a translaminar approach was used for the epidural glucocorticoid injection.

\section{Morbidity and Mortality Rates}

Morbidity and mortality rates were estimated for the multistate fungal meningitis outbreak by analyzing clinical data contained in the standardized case reports. ${ }^{17}$ After contacting more than $99 \%$ of 13,534 potential exposed patients by July 1, 2013, there were 749 reported cases with 61 deaths (8\%); 40 patients $(5 \%)$ had a stroke, and $229(31 \%)$ patients (of 728 case patients with additional data) had meningitis. Specimens from patients were tested for presence of fungi, and laboratory evidence confirmed the presence of
Exserohilum rostratum in specimens of 153 case patients (20\%). One injection (range, 1-6) from the implicated methylprednisolone acetate lots was administered among the case patients, with a median age of 64 years (range, 15-97 years) and median incubation period of 48 days (range, $0-249$ days) from the last injection to diagnosis.

\section{Morbidity and Mortality Reports: CDC}

The CDC released morbidity and mortality reports at the onset and throughout the multistate fungal meningitis outbreak affecting the 20 states. ${ }^{1,18,19}$ The CDC also maintained a website with real-time information on the outbreak investigation and additional information for patients, clinicians, and laboratories. ${ }^{20}$ As of October 23, 2013, the case count stood at 751 patients with 64 deaths, with no additional cases anticipated. A total of 233 patients had meningitis only, 325 patients had paraspinal/spinal infection only, 33 patients had peripheral joint infection only, 151 patients had both meningitis and paraspinal/spinal infection, 2 patients had paraspinal/spinal infection and peripheral joint infection, and 7 patients had strokes.

\section{DISCUSSION \\ Multisectoral Collaboration}

Multisectoral collaboration between the public health system at the local, state, and federal levels and the clinical system led to effective control of the fungal meningitis outbreak. The collaboration facilitated efficient epidemiological investigation, right from the detection of the index case and the infection cluster of the rare pathogen to rapid tracing of the outbreak source and followed by effective implementation of multiple response plans.

\section{Essential Services in Public Health}

The fungal meningitis outbreak response reflects both public health best practices and fidelity to the core responsibilities and essential services in public health, as promoted by the Institute of Medicine (IOM) and institutionalized in public 
health. ${ }^{21,22}$ The IOM reports on the future of public health conceptualized the core functions of public health assessment, policy development, and service assurances. The reports presented a multisectoral public health system framework of government public health agencies, working with multiple partners from the public and private sectors to improve population health. In the fungal meningitis outbreak response, public health assessment was accomplished through near real-time analysis of clinical and epidemiological data to trace the source of the outbreak. Policy development included written directives and procedures, with close attention to legalities and regulations, to prevent and control the spread of the fungal meningitis outbreak. Service assurances in the fungal meningitis outbreak response were accomplished through support of compliance efforts and enforcement throughout the multisectoral public health system.

\section{Public Health Implications}

We conducted a systematic review of the multisectoral outbreak response to the 2012-2013 multistate fungal meningitis epidemic in the United States from the perspectives of clinical response, outbreak investigation, and epidemiology. This systematic analysis of the well-coordinated outbreak response will assist in the preparedness of public health and clinical systems in the control and prevention of future outbreaks.

\section{Limitations}

The limitations of this systematic review were the exclusion of communication, such as phone calls and e-mails, between health departments at local, state, and federal levels and other stakeholders, such as clinicians, pharmacies, and insurance agencies, and regular follow-up communication by clinicians and local health departments with affected patients during the course of the fungal meningitis outbreak.

\section{About the Authors}

Department of Population Health Sciences, Virginia Tech, Blacksburg, Virginia (Drs Abbas and Redican and Ms Dorratoltaj); New River Health District, Virginia Department of Health, Christiansburg, Virginia (Dr O'Dell and Ms Bordwine); and Division of Infectious Diseases, Virginia Tech Carilion School of Medicine, Roanoke, Virginia (Dr Kerkering).

Correspondence and reprint requests to Kaja M. Abbas, Department of Population Health Sciences, Virginia Tech, Blacksburg, VA 24061 (e-mail: kaja.abbas@vt.edu).

\section{Funding}

This study was supported by the National Coordinating Center for Public Health Services and Systems Research and the National Institute of General Medical Sciences of the National Institutes of Health under Award Number R01GM109718. The funders had no role in study design, data collection and analysis, decision to publish, or preparation of the manuscript. The content is solely the responsibility of the authors and does not necessarily represent the official views of the funding agencies.

Published online: December 18, 2015.

\section{REFERENCES}

1. CDC. Multistate outbreak of fungal infection associated with injection of methylprednisolone acetate solution from a single compounding pharmacy United States, 2012. MMWR Morb Mortal Wkly Rep. 2012;61(41):839-842.

2. Kainer MA, Reagan DR, Nguyen DB, et al. Fungal infections associated with contaminated methylprednisolone in Tennessee. $N$ Engl J Med. 2012;367(23):2194-2203. http://dx.doi.org/10.1056/NEJMoa1212972.

3. CDC. Multistate fungal meningitis outbreak-interim guidance for treatment. MMWR Morb Mortal Wkly Rep. 2012;61(41).

4. Moher D, Liberati A, Tetzlaff J, Altman DG; The PRISMA Group. Preferred reporting items for systematic reviews and meta-analyses: the PRISMA Statement. PLoS Med. 2009;6(7):e1000097. http://dx.doi.org/ 10.1371/journal.pmed.1000097.

5. Kauffman CA, Pappas PG, Patterson TF. Fungal infections associated with contaminated methylprednisolone injections. $N$ Engl J Med. 2013;368(26):2495-2500. http://dx.doi.org/10.1056/NEJMra1212617.

6. Traynor K. Meningitis outbreak challenges hospital pharmacies. Am J Health Syst Pharm. 2012;69(23):2024-2026. http://dx.doi.org/10.2146/news120081.

7. Kerkering TM, Grifasi ML, Baffoe-Bonnie AW, et al. Early clinical observations in prospectively followed patients with fungal meningitis related to contaminated epidural steroid injections. Ann Intern Med. 2013;158(3): 154-161. http://dx.doi.org/10.7326/0003-4819-158-3-201302050-00568.

8. McKinney M. Battlefront. Michigan hospital at forefront in national fight against outbreak from tainted injections. Mod Healthc. 2012;42(47).

9. Kuehn BM. Hospital faces uncertainty as it copes with surge of patients with fungal meningitis. JAMA. 2013;309(3):219-221. http://dx.doi.org/ 10.1001/jama.2012.187705.

10. Malani AN, Vandenberg DM, Singal B, et al. Magnetic resonance imaging screening to identify spinal and paraspinal infections associated with injections of contaminated methylprednisolone acetate. JAMA. 2013;309(23):2465-2472. http://dx.doi.org/10.1001/jama.2013.6293.

11. Moudgal V, Singal B, Kauffman CA, et al. Spinal and paraspinal fungal infections associated with contaminated methylprednisolone injections. Open Forum Infect Dis. 2014;1(1):ofu022. http://dx.doi.org/10.1093/ofid/ofu022.

12. Pappas PG. Lessons learned in the multistate fungal infection outbreak in the United States. Curr Opin Infect Dis. 2013;26(6):545-550. http://dx. doi.org/10.1097/QCO.0000000000000013.

13. Bell BP, Khabbaz RF. Responding to the outbreak of invasive fungal infections: the value of public health to Americans. JAMA. 2013;309(9): 883-884. http://dx.doi.org/10.1001/jama.2013.526.

14. Todd B. Fungal meningitis outbreak. Am J Nurs. 2013;113(2):52-57. http://dx.doi.org/10.1097/01.NAJ.0000426691.81083.93.

15. Corvese K, Forlano L, Gibson L. A hybrid strategy for surveillance of individuals potentially exposed to contaminated methylprednisolone acetate-Virginia, 2012. J Public Health Manag Pract. 2013;19(4): 289-293. http://dx.doi.org/10.1097/PHH.0b013e318294e603.

16. Chiller TM, Roy M, Nguyen D, et al. Clinical findings for fungal infections caused by methylprednisolone injections. N Engl J Med. 2013;369(17):1610-1619. http://dx.doi.org/10.1056/NEJMoa1304879.

17. Smith RM, Schaefer MK, Kainer MA, et al. Fungal infections associated with contaminated methylprednisolone injections. $N$ Engl J Med. 2013;369(17):1598-1609. http://dx.doi.org/10.1056/NEJMoa1213978.

18. CDC. Spinal and paraspinal infections associated with contaminated methylprednisolone acetate injections - Michigan, 2012-2013. MMWR Morb Mortal Wkly Rep. 2013;62(19):377-381.

19. CDC. Notes from the field: department of Defense response to a multistate outbreak of fungal meningitis-United States, October 2012. MMWR Morb Mortal Wkly Rep. 2013;62(38):800-801.

20. CDC. Multistate outbreak of fungal meningitis and other infections. CDC website. http://www.cdc.gov/hai/outbreaks/meningitis.html. Accessed April 30, 2015.

21. Institute of Medicine. The Future of Public Health. Washington, DC: National Academy Press; 1988. http://iom.nationalacademies.org/Reports/ 1988/The-Future-of-Public-Health.aspx. Accessed November 10, 2015.

22. Institute of Medicine. The Future of the Public's Health in the 21st Century. Washington, DC: National Academy Press; 2002. http://iom.edu/Reports/ 2002/The-Future-of-the-Publics-Health-in-the-21st-Century.aspx. Accessed July 3, 2014. 